\title{
Abstract \\ The Value of 18F-FDG PET/contrast-enhanced CT in Detection of Tumor Thrombus
}

\section{Purpose}

The differentiation between tumor and bland thromboses is important as the management differs. Retrospectively, we aim to evaluate the utility of FDG PET in detecting and differentiating tumor from bland thromboses; and if FDG PET provides additional value to contrast-enhanced CT for tumor thrombus detection.

\section{Materials and Methods}

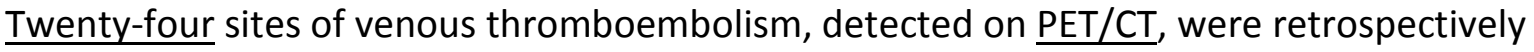
reviewed. Classification of type of thrombosis was based on histology and radiological follow-up. We evaluated the presence of contrast-enhanced CT findings that were suggestive of tumor thrombosis; sign of invasion, neovascularity and enhancement. Metabolic activity by means of SUVmax was measured by drawing ROI at the site of thrombosis. Mann-Whitney $\mathrm{U}$ test was used to compare the mean SUVmax between thromboses and internal references. We used ROC analysis to identify the optimal cut-off value of SUVmax for detection of tumor thrombosis.

\section{Results}

Twenty-four sites of venous thromboembolism were identified in 15 patients. All tumor thromboses demonstrated at least one positive sign on contrast-enhanced CT; whereas 33\% of bland thromboses had the same finding. The difference between tumor and bland thrombus SUVmax was statistically significant $(p<0.005)$. On ROC analysis, a cut-off of 
SUVmax 2.25 (sensitivity $78 \%$, specificity $100 \%$ ) was suggested to differentiate tumor from bland thrombosis.

\section{Conclusion}

$\underline{\mathrm{PET} / \mathrm{CT}}$ is able to differentiate tumor from bland thrombosis, with an optimal cut-off value of SUVmax 2.25. The metabolic information increases the diagnostic accuracy of tumor thrombus, and is a useful adjunct to the described features on contrast-enhanced CT.

Key Words: tumor thrombosis, PET, contrast-enhanced CT, 18F-FDG PET/CT, SUVmax

\section{Introduction}

Patients with underlying malignancy are predisposed to venous thromboembolism (VTE) secondary to the direct and indirect influences of malignancy on every element of Virchow's triad. With increasing use of imaging as part of disease assessment, there is increasing trend of incidental VTE detection. VTE can be divided into two broad categories- bland thrombus and tumor thrombus.

Tumor thrombus tends to be associated with solid tumor, including renal cell carcinoma ${ }^{1,2}$, Wilm's tumor ${ }^{3}$, testicular tumor ${ }^{4}$, adrenal cortical carcinoma, lymphoma ${ }^{5,6}$, pancreatic cancer $^{7}$, osteosarcoma ${ }^{8}$, Ewing's sarcoma ${ }^{9}$ and hepatocellular carcinoma ${ }^{10,11}$. Tumor thrombosis is an uncommon clinical entity for which the true incidence is unknown. It is estimated that $4-10 \%$ of renal cell carcinoma are associated with venous tumor thrombosis in the renal vein or inferior vena cava ${ }^{12}$ and a higher incidence of $20-30 \%$ are found in hepatocellular carcinoma ${ }^{10,11}$. The prognostic value of the presence of tumor 
thrombosis varies according to different tumor types; for example, portal vein tumor thrombosis in hepatocellular carcinoma is a poor prognostic factor, as surgical resection and trans-catheter arterial chemoembolization lack the desired therapeutic results ${ }^{10,11}$; while no impact on survival has been demonstrated in nephroblastoma with intracaval or atrial tumor thrombus ${ }^{13}$. However, consensus agrees that the primary treatment for tumor thrombosis should be surgical thrombectomy if possible, or chemo-radiation; while there is no role of anticoagulation therapy. Therefore, it remains important to be able to identify and differentiate tumor thrombosis from bland thrombosis to avoid the unnecessary use of anticoagulation, which carries the inherent risk of bleeding complications.

There are several contrast-enhanced CT features, previously described, which are suggestive of tumor thrombosis ${ }^{14,15}$. These include direct or contiguous invasion from tumor or metastasis (sensitivity of $32 \%$ and $62 \%$ respectively), neovascularity (sensitivity $43 \%$, specificity $100 \%$ ) and generalized intra-thrombus enhancement (sensitivity $83 \%)^{14}$. Dramatic venous expansion of portal vein in hepatocellular carcinoma has also been described ${ }^{14}$ and the cut-off mean diameter $\geqq 23 \mathrm{~mm}$ would give rise to $86 \%$ sensitivity and $100 \%$ specificity in CT detection of tumor thrombosis.

Though there are a handful of case reports ${ }^{16-25}$, there are only three small retrospective series ${ }^{26-28}$ that investigated the usefulness of 18 fluoro-deoxyglucose positron emission tomography (FDG PET) in detection of tumor thrombus; all concluded that FDG PET can differentiate tumor thrombosis from bland VTE based on SUV. Sharma et al ${ }^{28}$ suggested a cut-off value of maximum standardised uptake value (SUVmax) 3.63 with sensitivity yield of $72 \%$ and specificity of $90 \%$. None of these series have compared the accuracy of the two modalities, 18F-FDG PET and contrast-enhanced CT, in differentiating tumor from bland 
thrombus. We aim to investigate the utility of FDG PET in detecting and differentiating tumor from bland thrombus by means of SUVmax; and evaluate if FDG PET provides additional information to contrast-enhanced CT for tumor thrombus detection in a cohort of patients who underwent FDG PET/contrast-enhanced CT (ㅌT/CT).

\section{Materials and Methods}

The local institutional research and ethics review board approved this retrospective study.

\section{Patients}

Patients with VTE detected by PET/CT were identified through our database from March 2007 to May 2011. Demographic data, types of malignancy, clinical outcomes and follow-up imaging studies were recorded.

\section{PET/CT acquisition and interpretation}

Patient was required to fast 6 hour prior to $\underline{\mathrm{PET} / \mathrm{CT}}$ examination with glucose level below $144 \mathrm{mg} / \mathrm{dl}$ at the time of $18 \mathrm{~F}-\mathrm{FDG}$ injection. 18F-FDG dosage was weight-based: weight $(\mathrm{kg}) \mathrm{x}$ $0.13 \mathrm{mCi} . \underline{\mathrm{PET} / \mathrm{CT}}$ was acquired using dedicated PET/CT scanner (Discovery VCT; $64 \mathrm{MSCT}$, GE Healthcare Bio-Sciences Corp., Piscataway, NJ) 60 min following 18F-FDG injection. Contrast-enhanced CT protocol was as follows: $120 \mathrm{kVp}$; 200-400mA; field of view, 50cm; pitch $0.984: 1$; intravenous contrast medium $(1.5 \mathrm{ml} / \mathrm{kg})$ with injection rate of $2.0 \mathrm{ml} / \mathrm{sec}$; performed $70 \mathrm{sec}$ following intravenous contrast injection in the porto-venous phase. Attenuation correction for PET data using CT images was performed and images were reconstructed using an ordered-subset expectation maximization iterative algorithm (14 subsets and two iterations). All PET/CT studies were retrospectively reviewed by an 
experienced radiologist, trained to report $\underline{\mathrm{PET} / \mathrm{CT}}$ and blinded to the clinical or radiological follow-up data.

Sites of thromboses were identified on contrast-enhanced CT; CT features that were suggestive tumor thrombosis, i.e. direct or contiguous invasion from tumor, neovascularity within the thrombus and generalized intra-thrombus enhancement based on visual analysis were documented in both studied groups. Neovascularity and intra-thrombus enhancement were assessed qualitatively based on visual analysis as no non-contrast CT was performed as part of the PET/CT examination protocol. As venous expansion was only studied in the portal vein previously ${ }^{14}$, while there were various VTE sites involvement in our cohort and not exclusively confining to the portal veins, therefore venous expansion was excluded from statistical analysis.

Area of focal hypermetabolic activity corresponding to site of thrombus was measured using region of interest (ROI) by means of SUVmax; if no focal 18F-FDG uptake was identified, the ROI was manually placed over the site of thrombosis for SUVmax measurement (Figure 1). These were categorized into tumor thrombosis uptake (tSUVmax) and bland thrombosis uptake (bSUVmax) according the criteria set below based on histology or follow up imaging. Mediastinal blood pool uptake (mSUVmax) and background liver uptake (ISUVmax) were taken as internal references.

\section{Results interpretation}

Histological confirmation was taken as the gold standard when available. In the cohort without histological correlations, thrombus that resolved after anticoagulation therapy, was regarded as bland thrombus; whereas, progression of thrombus on follow-up imaging was regarded as indirect marker of tumor involvement. 


\section{Statistics}

Mean \pm standard deviation, and range were used as descriptive statistical analysis. The mean SUVmax of mediastinal blood pool, liver background, tumor and bland thromboses were compared using Mann-Whitney $U$ test for non-parametric data. Receiver operating characteristic curve (ROC curve) was produced to identify an optimal cut-off value of SUVmax to differentiate tumor thrombus from bland thrombus. All statistical analyses were performed using the statistical package SPSS for Windows (Version 11.0, SPSS, Chicago). A $p$-value of $<0.05$ was considered to indicate statistical significance.

\section{Results}

\section{Patients}

Forty patients were identified to have VTE from our database; only 15 patients had histological confirmation and imaging follow-up to determine the nature of VTE. Therefore 15 patients were included in the study analysis.

There were 7 males and 8 females with mean age of 62.7 years (median 58.5 years, range 46-76 years). All 15 patients had known primary malignancies and PET/CT scans were performed for staging/restaging or treatment evaluation. 5 patients had more than one site of VTE, giving rise to 24 sites of VTE for analysis. Demographic characteristics of the patients were tabulated in Table 1.

None of the above patients had concurrent inflammatory conditions at the time of PET/CT, therefore minimising the possibility of thrombophlebitis or pylephlebitis, which can give rise to increased metabolic activity ${ }^{29}$, as cause of hypermetabolic thrombus. 


\section{Classification of thromboses}

There were 18 sites of tumor thromboses and 6 sites of bland thromboses.

8 VTE sites had histological confirmation (Figure 2) while 16 sites had imaging follow-up in the form of $\underline{P E T / C T}, \mathrm{MRI}$, contrast enhanced CT (Figure 3) and US Doppler studies. The mean follow-up period was $143 \pm 104$ days (range 16-365 days).

\section{$\underline{\mathrm{PET} / \mathrm{CT}}$}

Among the 18 sites of tumor thromboses, direct invasion from tumors or metastases was present in 5 sites and contiguous spread in 10 sites; while the remaining 3 sites were remote from the tumors or metastases. On contrast-enhanced CT, 7 sites (39\%) demonstrated intra-thrombus neovascularity based on visual analysis and 9 sites (50\%) had inhomogeneous intra-thrombus enhancement (Table 1).

All 6 bland VTE sites (100\%) were remote from tumors or metastases, and none showed neovascularity. 2 bland thromboses demonstrated intra-thrombus enhancement based on visual analysis.

The mSUVmax and ISUVmax, as internal references, showed no statistical significance between tumor thrombosis and bland thrombosis ( $p=0.734$ and $p=0.865$ respectively). The differences between tSUVmax, and mSUVmax and ISUVmax were statistically significant (both $p<0.001$ ). The mean SUVmax for bland thrombosis group was $1.5 \pm 0.5$ (range 0.7-2.0) and the mean SUVmax for tumor thrombosis group was $4.5 \pm 4.5$ (range 1.0-14.8). The difference between bSUVmax and tSUVmax was statistically significant $(p=0.005)$ (Figure 4).

On ROC analysis, a cut-off SUVmax of 2.25 will yield $78 \%$ sensitivity and $100 \%$ specificity, in differentiating tumor thrombosis from bland thrombosis (Figure 5). 
If this is corrected against the mediastinal blood pool uptake (tSUVmax /mSUVmax), ROC analysis showed that a cut-off ratio of 1.58 will produce sensitivity of $83 \%$ and specificity $100 \%$ (Figure 6).

If the three outliers within the tumor thrombosis group were excluded (SUVmax 13.3, 14.2 and 14.8, see Figure 4), the ROC analysis produced similar results and the optimal cut-off SUVmax 2.25 remained unchanged (Figure 7).

In the 2 bland thromboses that exhibited intra-thrombus enhancement, the metabolic uptake was less than SUVmax 2.25 (SUVmax 1.9 and SUVmax 2.0 respectively). Therefore, FDG PET using SUVmax cut-off of 2.25 changed the contrast-enhanced CT diagnosis in $33 \%$ of bland thromboses.

\section{Discussion}

Previously described CT features that help to differentiate tumor from bland thrombus ${ }^{14,15}$ include direct invasion from tumor and neovascularity within the thrombus; whereas generalized thrombus enhancement and contiguous spread can be helpful to increase diagnostic confidence. Our results agreed with previous published data ${ }^{14}$. The lower incidence of intra-thrombus neovascularity found in our study could be explained by acquisition of CT images during the venous phase as neovascularity is easier to detect in early arterial phase of the contrast dynamic.

Previous three retrospective series ${ }^{26-28}$ using PET/ unenhanced CT have suggested that the metabolic uptake within the thrombus can be used to detect tumor thrombosis. Summary of these three studies were tabulated in Table 2. 
The FDG avidities in the primary tumors varied in this cohort comprising of heterogeneous types of tumors. Previous study has demonstrated positive correlation between the level of uptake in the primary tumor and tumor thrombus ${ }^{28}$. Therefore there is no surprise that the range of tSUVmax varied considerably.

ROC analysis identified that a cut-off of SUVmax 2.25 can be employed to differentiate tumor from bland thrombosis with sensitivity of $78 \%$, specificity $100 \%$ and accuracy $88 \%$. Using this cut-off value, all the bland thromboses that had CT features of intra-thrombus enhancement could be excluded, thus increasing the specificity of $\underline{P E T / C T}$. Tumor thrombosis commonly develops from direct or contiguous spread from the primary tumor or metastasis. The process could involve intra-luminal extension of tumor without invasion of the vascular endothelium or direct invasion into the segment of venous circulation. The presence of endothelial invasion tends to preclude successful surgical thrombolectomy and the risk of tumor spread is higher. This may partly explain why the presence of tumor thrombosis has different prognostic impact on different carcinomas. For example hepatocellular carcinoma commonly invades the portal vein producing tumor thrombosis, and is associated with poorer outcome. In renal cell carcinoma, tumor thrombus can propagate into the renal vein and inferior vena cava without invasion of the vascular endothelium. Therefore, if the tumor thrombus can be successfully resected, it will not be of any prognostic significance in patient's survival ${ }^{30}$. In our study, contrast-enhanced CT was performed sequentially with FDG PET. Although there has been concern regarding the impact of IV contrast on CT-based attenuation correction, especially in regions of dense IV contrast ${ }^{31}$; this study assessed regions of relatively lower IV contrast concentration in the venous circulation (performed at 70 
seconds after IV contrast injection), and therefore is not expected to significantly affect the CT-based attenuation coefficient ${ }^{32}$.

This study has several limitations. The study includes a heterogeneous group of tumors which have different FDG avidities and hence, the varied FDG uptake in the tumor thrombus. The lack of true gold standard in some of the cases may also introduce bias into the analysis of this study, as histological confirmation was only limited to 8 VTE sites. The sample number is small, although the results were of statistical significance despite the small numbers. We agree with previous authors ${ }^{28}$ that larger study is required and ideally with histological validations of the diagnoses.

\section{Conclusion}

The measurement of SUVmax on FDG PET is of additional value to contrast-enhanced CT in differentiating tumor thrombosis from bland thrombosis, especially in excluding the presence of tumor thrombosis using SUVmax cut-off value of 2.25. This information will enhance the accuracy of scan interpretation especially with the increasing use of diagnostic contrast-enhanced CT together with FDG PET, as a 'one-stop shop'.

\section{Acknowledgement}

The authors would like to thank Mr. Huang, BS. for his assistance in the statistical analysis. 


\section{References}

1. Moinzadeh A, Libertino JA: Prognostic significance of tumor thrombus level in patients with renal cell carcinoma and venous tumor thrombus extension. Is all T3b the same? J Urol. 2004;171:598-601.

2. Sweeney P, Wood CG, Pisters LL, et al: Surgical management of renal cell carcinoma associated with complex inferior vena caval thrombi. Urol Oncol. 2003;21:327-333.

3. Shamberger RC, Ritchey ML, Haase GM, et al. Intravascular extension of Wilms tumor. Ann Surg. 2001;234:116-121.

4. Donohue JP, Thornhill JA, Foster RS, et al: Resection of the inferior vena cava or intraluminal vena caval tumor thrombectomy during retroperitoneal lymph node dissection for metastatic germ cell cancer: indications and results. J Urol. 1991;146:346-349.

5. Natsuizaka M, Kudo M, Suzuki M, et al: Diffuse large B-cell lymphoma with massive portal vein tumor thrombosis in a patient with alcoholic cirrhosis: a case report and literature review. Intern Med. 2009;48:805-808.

6. Tripathi M, D'Souza M, Jain J, et al: Sagittal sinus tumor thrombosis in a case of NHL demonstrated on F-18 FDG PET/CT. Clin Nucl Med. 2011;36:252-254.

7. Nguyen BD: Pancreatic neuroendocrine tumor with portal vein tumor thrombus: PET demonstration. Clin Nucl Med. 2005;30:628-629.

8. Garcia ND, Morasch MD, Sam AD, 2nd, et al: Inferior vena cava thrombus removal using hypothermic circulatory arrest in two patients with osteosarcoma. Ann Vasc Surg. 2003;17:686-689. 
9. Karnes RJ, Gettman MT, Anderson PM, et al: Primitive neuroectodermal tumor (extraskeletal Ewing's sarcoma) of the kidney with vena caval tumor thrombus. J Urol. 2000;164:772.

10. Prospective validation of the CLIP score: a new prognostic system for patients with cirrhosis and hepatocellular carcinoma. The Cancer of the Liver Italian Program (CLIP) Investigators. Hepatology. 2000;31:840-845.

11. Park KW, Park JW, Choi JI, et al: Survival analysis of 904 patients with hepatocellular carcinoma in a hepatitis B virus-endemic area. J Gastroenterol Hepatol. 2008;23:467-473.

12. Marshall FF, Dietrick DD, Baumgartner WA, et al: Surgical management of renal cell carcinoma with intracaval neoplastic extension above the hepatic veins. J Urol. 1988;139:1166-1172.

13. Ritchey ML, Kelalis PP, Breslow N, et al: Intracaval and atrial involvement with nephroblastoma: review of National Wilms Tumor Study-3. J Urol. 1988;140:1113-1118.

14. Tublin ME, Dodd GD, 3rd, Baron RL: Benign and malignant portal vein thrombosis: differentiation by CT characteristics. AJR. Am J Roentgenol. 1997;168:719-723.

15. Shah ZK, McKernan MG, Hahn PF, et al: Enhancing and expansile portal vein thrombosis: value in the diagnosis of hepatocellular carcinoma in patients with multiple hepatic lesions. AJR. Am J Roentgenol. 2007;188:1320-1323.

16. Sizemore AW, Jacobs MP, Mantil JC, et al: FDG uptake in inferior vena cava tumor thrombus from renal cell carcinoma on positron emission tomography. Clin Nucl Med. 2007;32:309-311. 
17. Snow D, Cohen D, Chapman WC, et al: Positron emission tomography enhancing tumor thrombus in patient with renal cell carcinoma. Urology. 2009;73:270-271.

18. Probst S, Seltzer A, Chachoua A, et al: Azygos venous tumor thrombus from renal cell carcinoma detected by F-18 FDG PET/CT. Clin Nucl Med. 2010;35:832-833.

19. Roldan-Valadez E, Ortega-Lopez N, Valdivieso-Cardenas G, et al: (18)F-FDG PET/CT for discrimination between tumor extension and blood thrombus in pancreatic adenocarcinoma associated with portal vein thrombosis. Rev Esp Med Nucl. 2008;27:40-44.

20. Hanajiri K, Mitsui H, Maruyama T, et al: 18F-FDG PET for hepatocellular carcinoma presenting with portal vein tumor thrombus. J Gastroenterol. 2005;40:1005-1006.

21. Sun L, Wu H, Pan WM, et al: Positron emission tomography/computed tomography with (18)F-fluorodeoxyglucose identifies tumor growth or thrombosis in the portal vein with hepatocellular carcinoma. World J Gastroenterol. 2007;13:4529-4532.

22. Gupta P, Kramer EL, Ponzo F: FDG uptake in tumor thrombus in inferior vena cava from rectal cancer on positron emission tomography. Clin Nucl Med. $2005 ; 30: 342-343$.

23. Kaida $\mathrm{H}$, Ishibashi $\mathrm{M}$, Kurata $\mathrm{S}$, et al: Tumor thrombus in the inferior vena cava from colon cancer detected by 18F-FDG-PET. Ann Nucl Med. 2007;21:185-188.

24. Strobel K, Steinert HC, Bhure U, et al: Tumour thrombus in the superior vena cava from anaplastic carcinoma of the thyroid: FDG-PET/CT imaging findings. Eur J Nucl Med Mol Imaging. 2007;34:813. 
25. Tripathi M, Sharma R, Jaimini A, et al: Metastatic follicular carcinoma of the thyroid with tumor thrombus in the superior vena cava and right brachiocephalic and internal jugular veins: FDG-PET/CT findings. Clin Nucl Med. 2008;33:426-428.

26. Lai $\mathrm{P}$, Bomanji JB, Mahmood S, et al: Detection of tumour thrombus by 18F-FDG-PET/CT imaging. Eur J Canc Prev. 2007;16:90-94.

27. Davidson T, Goitein O, Avigdor A, et al: 18F- FDG-PET/CT for the diagnosis of tumor thrombosis. Isr Med Assoc J. 2009;11:69-73.

28. Sharma P, Kumar R, Jeph S, et al: 18F-FDG PET-CT in the diagnosis of tumor thrombus: can it be differentiated from benign thrombus? Nucl Med Commun. 2011;32:782-788.

29. Miceli $M$, Atoui $R$, Walker $R$, et al: Diagnosis of deep septic thrombophlebitis in cancer patients by fluorine-18 fluorodeoxyglucose positron emission tomography scanning: a preliminary report. J Clin Oncol. 2004;22:1949-1956.

30. Gonzalez J, Angulo J, Ciancio G: Surgical management of renal cell cancer with tumor thrombus through an exclusive transabdominal approach. Arch Esp Urol. 2011;64:297-309.

31. Mawlawi O, Erasmus JJ, Munden RF, et al: Quantifying the effect of IV contrast media on integrated PET/CT: clinical evaluation. AJR. Am J Roentgenol. 2006;186:308-19.

32. Bacharach SL: PET/CT attenuation correction: breathing lessons. J Nucl Med. 2007;48:677-679. 


\section{Legends}

Table 1. Demographic details with sites of thromboses, presence of positive signs on contrast-enhanced CT suggestive of tumor thrombosis, metabolic uptake, categorization of types of thrombosis and confirmatory evidence. Pink rows: tumor thrombi; Light green rows: bland thrombi; Ca: carcinoma; PV: portal vein; IVC: inferior vena cava; LA: left atrium; SMV: superior mesenteric vein; IJV: internal jugular vein; SVC: superior vena cava; PE: pulmonary embolism; IMV: inferior mesenteric vein; ceCT: contrast-enhanced CT; D: direct invasion; C: contiguous spread; R: remote from tumor; N: neovascularity; E: intra-thrombus enhancement; mSUVmax: mediastinal SUVmax; ISUVmax: liver SUVmax; FU: follow-up

Table 2. Summary of three retrospective studies describing the usefulness of FDG PET in detecting tumor thrombus and their relevant findings.

Figure 1.42 year-old female with ovarian carcinoma and left common iliac vein thrombosis. Coronal contrast-enhanced $\mathrm{CT}$ and coronal fused $\underline{\mathrm{PET} / \mathrm{CT}}$ with manually placed ROI over the site of bland thrombosis for SUVmax measurement.

Figure 2a. 58 year-old lady with left renal cell carcinoma. Coronal fused PET/CT demonstrates hypermetabolic left RCC (red solid arrow) with hypermetabolic tumor thrombus (white solid arrow) extending into the left renal vein and IVC. On visual inspection, the tumor thrombus has higher metabolic activity compared to background liver and mediastinal blood pool.

Figure 2b. 58 year-old lady with left renal cell carcinoma. Axial contrast enhanced CT demonstrates heterogeneous filling defect in the left renal vein, extending into the IVC (white solid arrow), which are distended, compatible with venous thromboses. 
Figure 3a. 58 year-old man with primary rectal leiomyosarcoma. Maximum intensity projection demonstrates multiple hypermetabolic nodal, liver and osseous metastases. Figure 3b. 58 year-old man with primary rectal leiomyosarcoma. Fused coronal PET/CT demonstrates linear hypermetabolic left common femoral vein tumor thrombus (red solid arrow), SUVmax 3.4 .

Figure 3c. 58 year-old man with primary rectal leiomyosarcoma. Corresponding coronal contrast enhanced CT demonstrates filling defect in the left common femoral vein which is distended, consistent with venous thrombosis.

Figure 4. Box-plot demonstrates the SUVmax of bland thrombosis group and tumor thrombosis group. The difference between the two groups are statistically significant, $p=0.005$ (Mann-Whitney U Test).

Figure 5. ROC curve produces a cut-off value of SUVmax 2.25 , with $78 \%$ sensitivity and $100 \%$ specificity (area under curve, AUC index 0.889) to differentiate tumor from bland thromboses.

Figure 6. ROC curve produces a cut-off ratio tSUVmax/mSUVmax of 1.58 , with $83 \%$ sensitivity and $100 \%$ specificity (AUC index 0.88 ) to differentiate tumor from bland thromboses.

Figure 7. ROC curve excluding the three outliers, produces the same cut-off SUVmax of 2.25, with $83 \%$ sensitivity and $100 \%$ specificity (AUC index 0.876 ) to differentiate tumor from bland thromboses. 
Figure 1

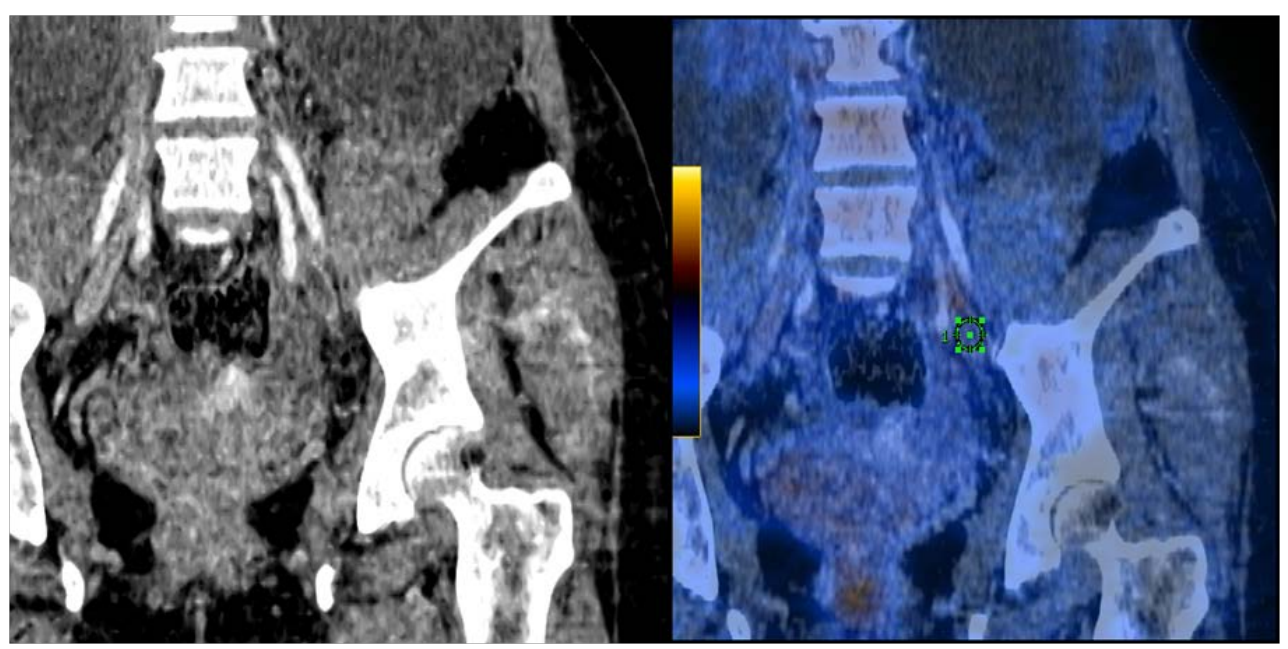

Figure 2a.

Figure 2b.
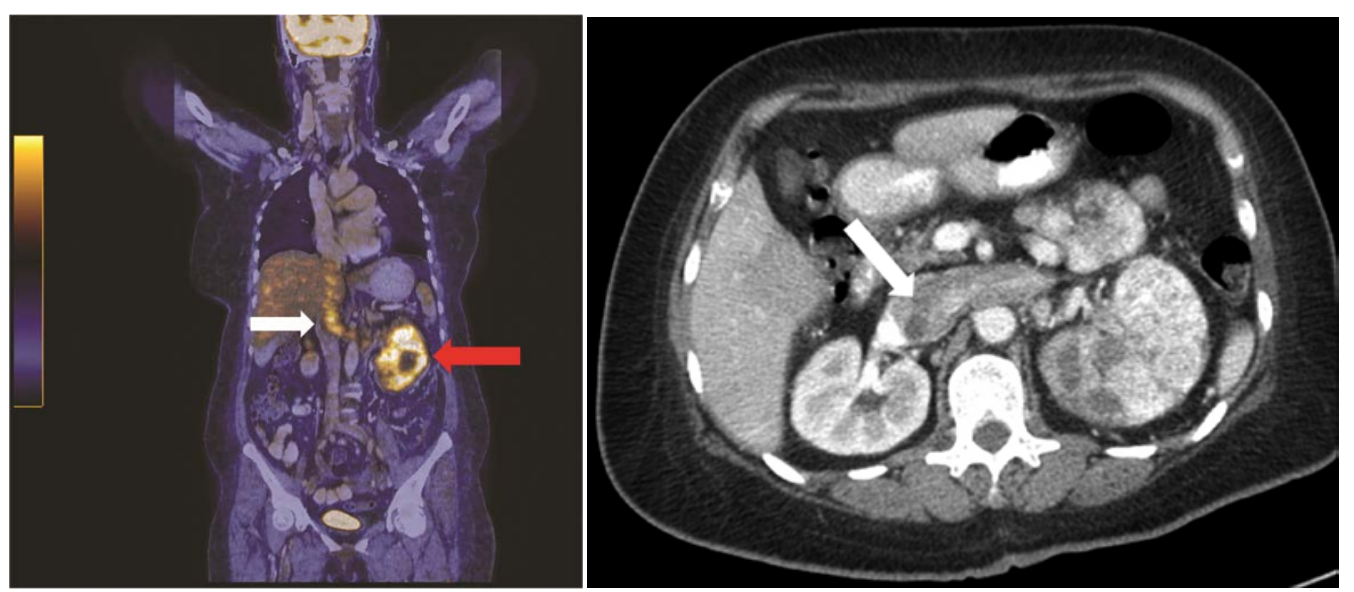

Figure 3a.

Figure $3 b$.
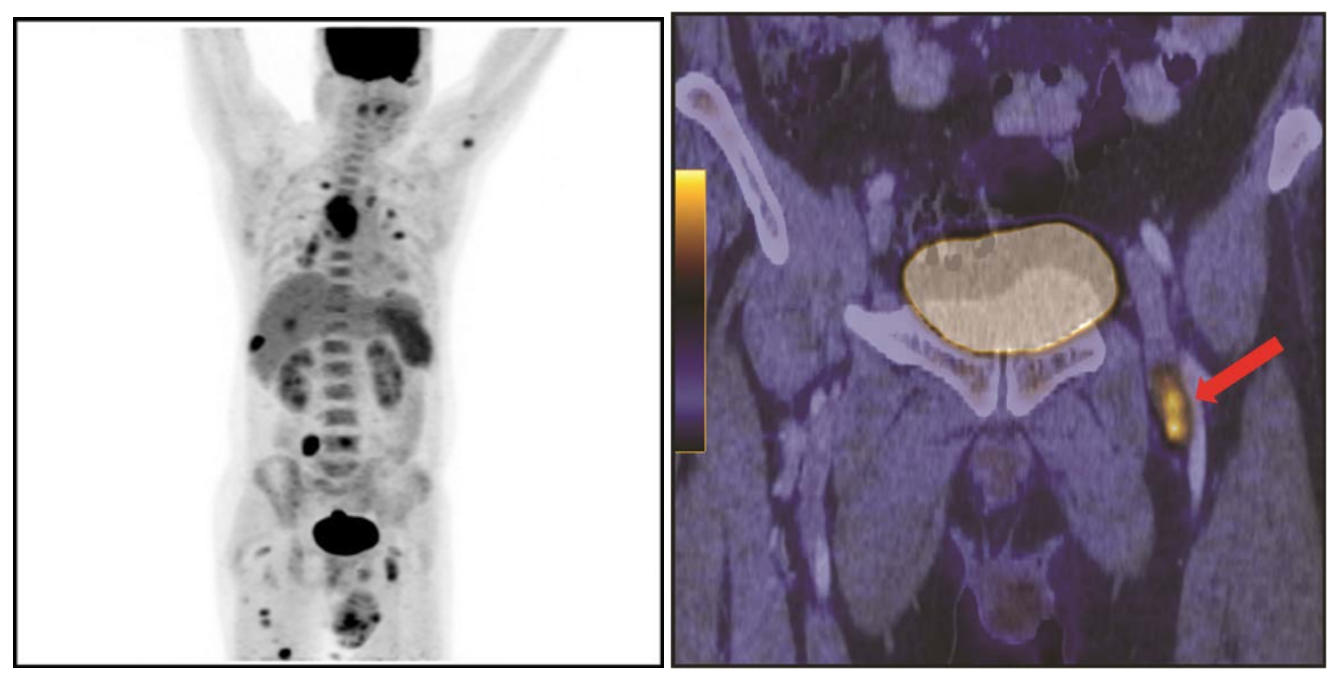
Figure 3c.

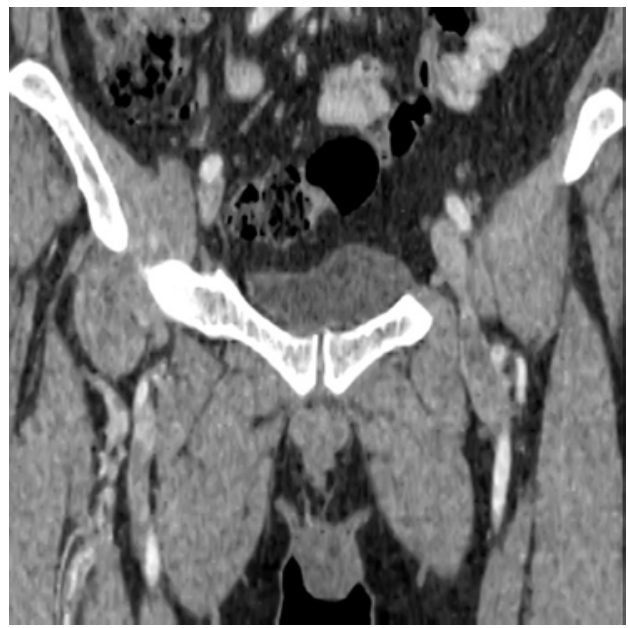

Figure 5.
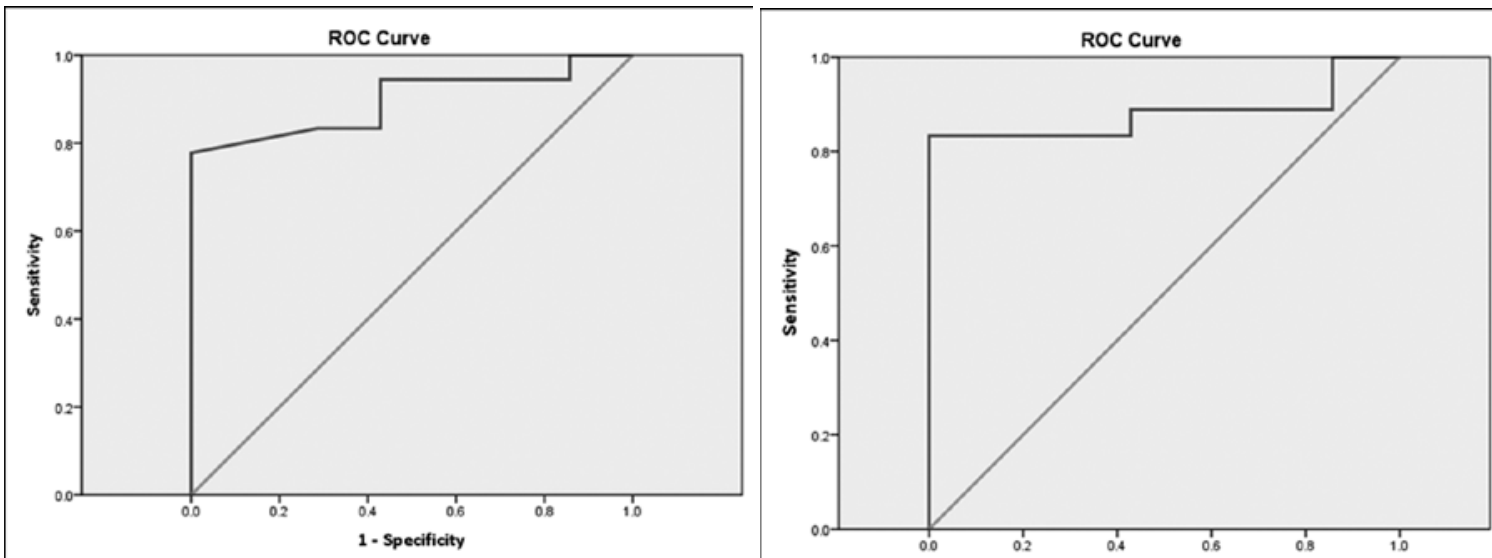

Figure 7.

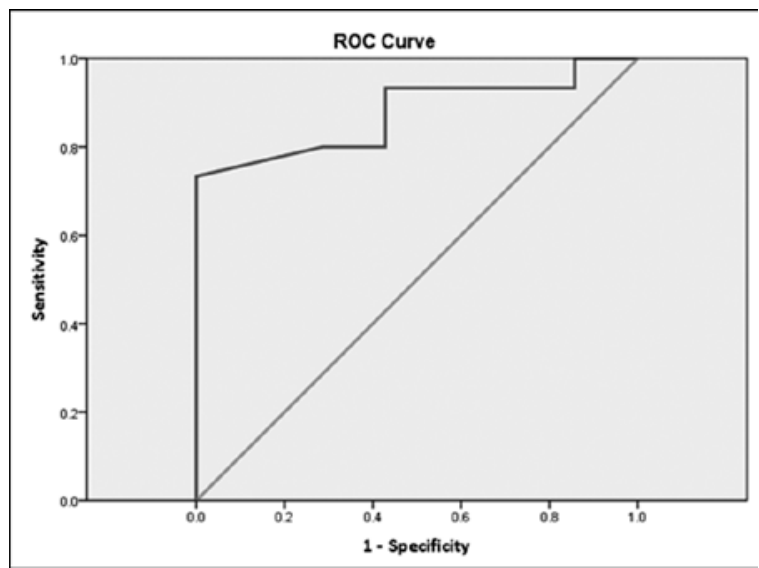

